\title{
Quantum Chromodynamic Quark Model Study of Hadron and Few Hadron Systems
}

\author{
Progress Report
}

July 1990 - November 1990

\author{
Chueng-Ryong Ji \\ Department of Physics \\ North Carolina State University \\ Raleigh, North Carolina 27695-8202
}

\section{October 1990 \\ DISCLAIMER}

\begin{abstract}
This report was prepared as an account of work sponsored by an agency of the United States Government. Neither the United States Gnvernment nor any agency thereof, nor any of their employees, makes any warranty, express or implied, or assumes any legal liability or responsibility for the accuracy, completeness, or usefulness of any information, apparatus, product, or process disclosed, or represents that its use would not infringe privately owned rights. Reference herein to any specific commercial product, process, or service by trade name, trademark, manufacturer, or otherwise does not necessarily constitute or imply its endorsement, recommendation, or favoring by the United States Government or any agency thereof. The views and opinions of authors expressed herein do not necessarily state or reflect those of the United States Government or any agency thereof.
\end{abstract}

Prepared for the U. S. Department of Energy under Grant Number DE-FG05-90ER40589. 
This report details research progress and results obtained during the five month period July 1, 1990 to November 30, 1990. The research project, entitled "Quantum Chromodynamic Quark Model Study of Hadron and Few Hadron Systems", is supported by grant DE-FG05-90ER40589 between North Carolina' State University and the United States Department of Energy. In compliance with grant requirements the Principal Investigator, Professor Chueng-Ryong Ji, has conducted a research program addressing theoretical investigations of hadron structure and reactions using quantum chromodynamic quark models. This Principal Investigator has devoted $50 \%$ of his time during the academic year and $100 \%$ of his time in the summer. This percent effort will continue for the remaining period of the grant. The new, significant research results are briefly summarized in the following sections. Recent progress has been reported in the renewal/continuation grant proposal just submitted to the Department of Energy. Finally, full, detailed descriptions of completed work can be found in the project publications which are listed at the end of this progress report. 
A new progress has been made in our long term, ambitious program dedicated to developing more realistic quantum chromodynarnic quark models. Our most recent investigation focused upon finding a severe constraint in building QCD-oriented Bethe-Salpeter model of qā hadrons. We found that the gauge invariance (or current conservation) plays an important role of constraint to build or test a hadron model. As an example, we explicitly showed that the hadron model suggested by Mitra and his collaborators does not satisfy the gauge invariance when the calculation does not include an odd number of $\gamma_{5}$ matrices and such violation leads to a poor approximation for some processes by severely truncating the problem. A portion of this work has been recently published in Phys. P.ev. Lett.

In addition to studies on constraints, the actual development of a gauge and Lorentz invariant QCD inspired model has been reviewed to assist the comparison between theoretical calculations and accurate experimental data anticipated from CEBAF. This investigator presented graduate summer school lectures (HUGS at CEBAF) this year. 


\section{QCD Pinthomenology}

During the past funding period several significant results were obtained in our QCD phenomenology project. In a recent leading-order perturbative $\mathrm{QCD}$ analysis of the nucleon Dirac form factor, we have shown that it is possible to fit the data in the range of momentum transfer square $10<\mathrm{Q}^{2}<30 \mathrm{GeV}^{2}$, by evaluating the argument of $\mathrm{QCD}$ running coupling constant $\alpha_{\mathrm{s}}\left(\widetilde{\mathrm{Q}}^{2}\right)$ at the exact gluon momentum transfer for each of the diagrams contributing to the leading-order process. We extended the same considerations to the pion and kaon form factors and their pair production in two-photon collisions, $\gamma \rightarrow \pi^{+} \pi^{-}, \gamma \rightarrow \mathrm{K}^{+} \mathrm{K}^{-}$, and compare our results with experimental data. Our aim was to apply the same method and QCD running coupling constant adopted in the previous nucleon-form-factor calculation to these pion- and kaon-induced processes and to investigate whether the same method and cutoff value can give a consistent agreement with the available experimental data. The cutoff value of the frozen coupling constant used in our analysis is consiscent with the theoretical estimation presented by Cornwall. A paper describing full details is now in press for publication in Phys. Rev. D. 
New results were obtained in the project of relativistic quark model calculations of hadron properties. Using a simplẹ relativistic quark model suggested by Dziembowski et al., we studied the open flavored pseudoscalar meson, i.e., $Q \bar{q}$, where $Q$ is $\mathbf{s}$ or $\mathbf{c}$ and $q$ is $u$ or d. Our analysis, which is a straight forward extension of their previous treatment for the pion, focuses on the kaon to provide further tests of this approach. Our results support the utility of this model as the predictions for the kaon charge radius $\left(r_{\mathrm{K}}^{2}\right)^{1 / 2}$, the kaon form factor $\mathrm{F}_{\mathrm{K}}$, and the decay constant $f_{\mathrm{K}}$ compare favorably with experimental data. A paper describing full details has been recently published in Phys. Rev. D. 


\section{PAPERS PUBLISHED IN PRESS IN THE REPORT PERIOD}

Refereed Journal Publication

1. "Perturbative QCD Analysis of the Pion and Kaon Form Factors and Pair Production in Photon-Photon Collisions Using a Frozen Coupling Constant", C.-R. Ji and F. Amiri, Phys. Rev. D42, in press.

2. "Comment on 'Understanding Electroweak Couplings of the Pion as a qa Composite'", C.R. Ji and S. R. Cotanch, Phys. Rev. Lett. 64, 1848 (1990).

3. "Simple Relativistic Quark Model Analysis of Flavored Pseudoscalar Mesons", C.-R. Ji and S. R. Cotanch, Phys. Rev. D41, 2319 (1990).

4. "Crossing Consistent Analysis of Kaon Photoproduction and Radiative Capture", R. Williams, C.-R. Ji and S. R. Cotanch, Phys. Rev. D41, 1449 (1990).

Invited Lectures

1. "Investigating Light-Cone QCD at CEBAF", C.-R. Ji, in the Fifth HUGS at CEBAF Summer School, Newport News, May 29 - June 16, 1990.

2. "QCD Phenomenology on Hadron Form Factors", C.-R. Ji, in Proceedings of the Summer School of Computational Atomic and Nuclear Physics, edited by C. Bottcher, M. R. Strayer and J. B. McGrory, World Scientific (Singapore), pp. 401-411, 1990. 

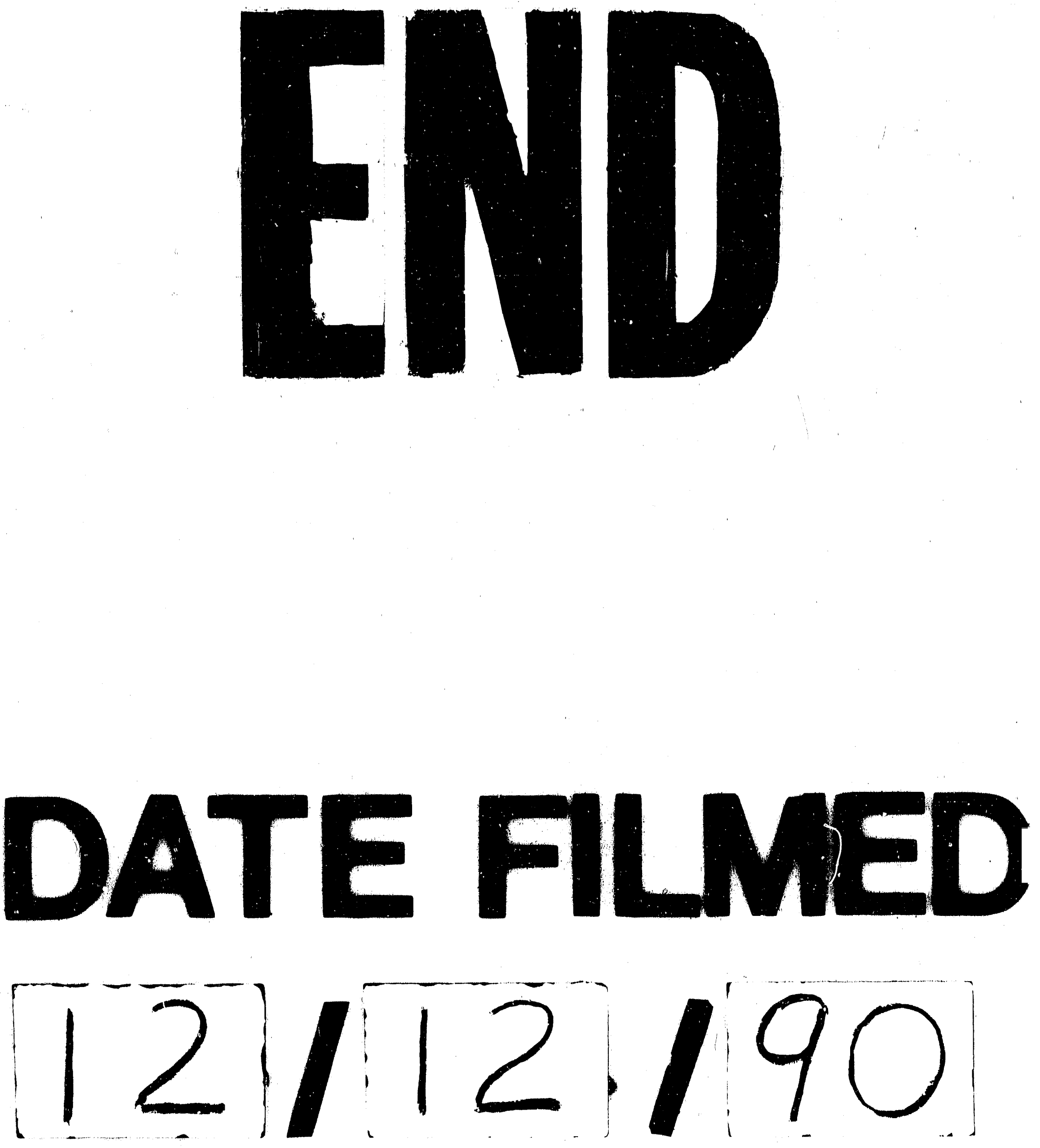


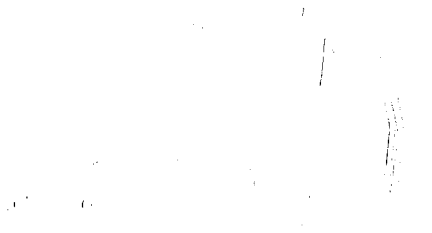

